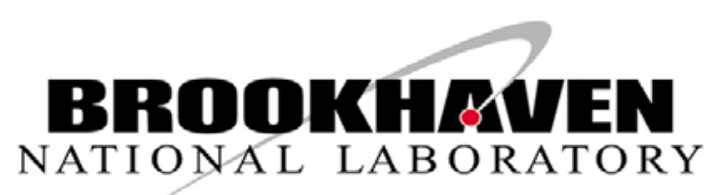

BNL-113509-2017-JA

\title{
Facile Synthesis of Silver Nanocubes with Sharp Corners and Edges in an Aqueous Solution
}

\author{
Shan Zhou, Jianhua Li, Kyle D. Gilroy, Jing Tao, \\ Chunlei Zhu, Xuan Yang, Xiaojun Sun, and Younan Xia
}

Submitted to ACS Nano

November 2016

\begin{abstract}
Condensed Matter Physics and Materials Science Department
Brookhaven National Laboratory
\end{abstract}

\author{
U.S. Department of Energy \\ USDOE Office of Science (SC), \\ Basic Energy Sciences (BES) (SC-22)
}




\section{DISCLAIMER}

This report was prepared as an account of work sponsored by an agency of the United States Government. Neither the United States Government nor any agency thereof, nor any of their employees, nor any of their contractors, subcontractors, or their employees, makes any warranty, express or implied, or assumes any legal liability or responsibility for the accuracy, completeness, or any third party's use or the results of such use of any information, apparatus, product, or process disclosed, or represents that its use would not infringe privately owned rights. Reference herein to any specific commercial product, process, or service by trade name, trademark, manufacturer, or otherwise, does not necessarily constitute or imply its endorsement, recommendation, or favoring by the United States Government or any agency thereof or its contractors or subcontractors. The views and opinions of authors expressed herein do not necessarily state or reflect those of the United States Government or any agency thereof. 


\title{
Facile Synthesis of Silver Nanocubes with Sharp Corners and Edges in an Aqueous Solution
}

\author{
Shan Zhou, ${ }^{\dagger}$ Jianhua Li, ${ }^{\ddagger}$ Kyle D. Gilroy, ${ }^{\ddagger}$ Jing Tao, ${ }^{\circledR}$ Chunlei Zhu, ${ }^{\ddagger}$ \\ Xuan Yang, ${ }^{\ddagger}$ Xiaojun Sun, ${ }^{\S}$ and Younan $\mathrm{Xia}^{*}{ }^{+}$, ,
}

${ }^{\dagger}$ School of Chemistry and Biochemistry, Georgia Institute of Technology, Atlanta, Georgia 30332, United States

‡The Wallace H. Coulter Department of Biomedical Engineering, Georgia Institute of Technology and Emory University, Atlanta, Georgia 30332, United States

"Condensed Matter Physics and Materials Science Department, Brookhaven National Laboratory, Upton, New York 11973, United States

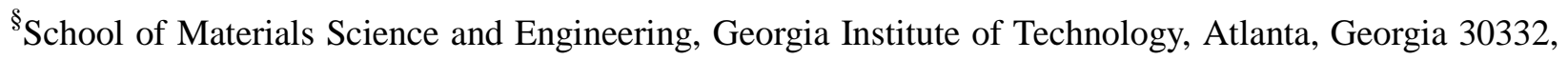
United States

*Address correspondence to younan.xia@bme.gatech.edu 
ABSTRACT. It remains a challenge to synthesize Ag nanocubes in an aqueous system although the polyol process has been successfully adopted more than one decade ago. Here we report an aqueous method for the synthesis of Ag nanocubes with an average edge length of 35-95 nm. It involves the formation of $\mathrm{AgCl}$ octahedra by mixing $\mathrm{CF}_{3} \mathrm{COOAg}$ with cetyltrimethylammonium chloride (CTAC), followed by the nucleation and growth of Ag nanocrystals in the presence of ascorbic acid (AA) and $\mathrm{FeCl}_{3}$. The $\mathrm{Fe}^{3+} / \mathrm{Fe}^{2+}$ redox pair is responsible for the removal of multiply twinned seeds through oxidative etching. The $\mathrm{Cl}^{-}$ions play two critical roles in the nucleation and growth of Ag nanocubes with a single-crystal structure. Firstly, the $\mathrm{Cl}^{-}$ions react with $\mathrm{Ag}^{+}$ions to generate nanometer-sized $\mathrm{AgCl}$ octahedra in the initial stage of a synthesis. In the presence of room light and a proper reducing agent such as $\mathrm{AA}$, the $\mathrm{AgCl}$ can be reduced to generate $\mathrm{Ag}_{\mathrm{n}}$ nuclei, followed by their evolution into single-crystal seeds and then $\mathrm{Ag}$ nanocrystals. Secondly, the $\mathrm{Cl}^{-}$ions can act as a specific capping toward the $\mathrm{Ag}(100)$ surface, enabling the formation of Ag nanocubes with sharp corners and edges. Based on the results from a set of time-elapse studies and control experiments, we formulate a plausible mechanism to account for the formation of Ag nanocubes, which resembles the formation and development of latent image centers in silver halide grains in the photographic process. In addition to the demonstration of a greener and economically more favorable method than the polyol process, this work also offers insights into the design of aqueous protocols for the synthesis of silver nanocrystals with controlled shapes.

Keywords: silver, nanocube, aqueous method, one-pot synthesis, silver chloride 


\section{Introduction}

Silver nanocrystals have found widespread use in a range of applications involving localized surface plasmon resonance (LSPR), surface-enhanced Raman scattering (SERS), metal-enhanced fluorescence, and catalysis. ${ }^{1,2}$ For most of these applications, it is critical to have a tight control over the shape taken by nanocrystals in order to maximum their performance. Over the past decade, Ag nanocrystals have been successfully synthesized with a large number of diversified shapes, including spheres, ${ }^{3}$ cubes, ${ }^{2,4-8}$ bars, ${ }^{9}$ tetrahedra, ${ }^{10}$ octahedra, ${ }^{11}$ decahedra, ${ }^{12}$ right bipyramids, ${ }^{13}$ and pentagonal rods/wires. ${ }^{14}$ Among these examples, nanocubes are of great interest because they can serve as sacrificial templates to fabricate Au-based nanocages with tunable LSPR peaks in the near-infrared region, ${ }^{15,16}$ and as outstanding substrates for optical sensing and SERS detection. ${ }^{17}$ Among all synthetic methods that have been developed for the synthesis of Ag nanocubes, polyol reduction is probably the most widely used and most successful approach. The polyol process uses alcohols with more than one hydroxyl groups as both the solvent and reductant (or a precursor to the reductant). The most extensively used polyols include ethylene glycol (EG) and diethylene glycol (DEG). After more than one decade of refinement, polyol reduction can now be applied to synthesize Ag nanocubes with edge lengths over a very broad range of $13-400 \mathrm{~nm} .^{2,4-8}$

Despite its great success in the synthesis of Ag nanocrystals, polyol reduction also has a few drawbacks. For example, the synthesis is very sensitive to impurities (such as trace amounts of $\mathrm{Cl}^{-}$and $\mathrm{Fe}^{2+} / \mathrm{Fe}^{3+}$ ions), water content, and the amount of $\mathrm{O}_{2}$, making the synthesis sometimes difficult to replicate. At the same time, the Ag nanocubes synthesized using the polyol process

tend to be rounded at corners and edges, ${ }^{2,15}$ which can be attributed to three major reasons: $i$ ) the relatively high diffusivity of $\mathrm{Ag}$ atoms at an elevated temperature (typically, in the range of $140-160{ }^{\circ} \mathrm{C}$ ) needed for activating the reduction power of a polyol; ii) the enhanced oxidative etching of corners and edges at an elevated temperature, and iii) the inadequacy of poly(vinyl pyrrolidone) (PVP) in passivating and protecting the $\{100\}$ side faces as a result of their long chains. In addition, the use of an organic solvent and the requirement of a relatively high temperature also make the protocol environmentally less friendly and economically unattractive. These drawbacks could be eliminated by developing a water-based route. In this case, an additional reducing agent has to be introduced because $\mathrm{H}_{2} \mathrm{O}$ lacks the power to reduce $\mathrm{Ag}^{+}$ions into the elemental form. In principle, an aqueous synthesis can be carried out at a much lower 
temperature than the polyol process, facilitating the formation and preservation of the sharp edges and corners on Ag nanocubes. The exclusion of polyols also solves the irreproducibility problem associated with trace impurities. ${ }^{18}$ Furthermore, a water-based synthesis is anticipated to be greener and environmentally more friendly, offering more flexibility and convenience for execution (e.g., outside the fume hood).

Although aqueous protocols have been explored by a number of groups for the seed-mediated growth of Ag nanocrystals, ${ }^{11,19-24}$ there are very few reports on the synthesis of Ag single-crystal nanocubes using a one-pot, aqueous system. The conditions for seed formation and the following growth step are often very different, making it more challenging to achieve precise control in one-pot synthesis than in seed-mediated growth. After extensive literature search, we were only able to find one report on the one-pot, aqueous method, in which Ag nanocubes with edge lengths of 55 to $72 \mathrm{~nm}$ were prepared. ${ }^{25,26}$ This method, however, involved the use of hydrothermal conditions at $120{ }^{\circ} \mathrm{C}$, and it is still a challenge to monitor and analyze the growth of nanocrystals, especially in the early stages of a synthesis, in such a closed system. In addition, this method used a hazardous precursor based on $\mathrm{Ag}\left(\mathrm{NH}_{3}\right)_{2} \mathrm{OH}$, an unstable compound that might decompose to generate explosive silver azide when handled improperly. ${ }^{27}$

Herein we reported a facile method for the synthesis of Ag nanocubes in an aqueous system. The Ag nanocubes could be made with sharp corners and edges by employing $\mathrm{Cl}^{-}$ions as a specific capping agent for the $\{100\}$ facets and conducting the synthesis at a relatively low temperature of $60{ }^{\circ} \mathrm{C}$. By mixing $\mathrm{CF}_{3} \mathrm{COOAg}$ with $\mathrm{CTAC}$ in an aqueous solution, $\mathrm{AgCl}$ nanoscale octahedra were formed in the early stage, which then served as a source to elemental Ag. In the presence of both room light and a reducing agent such as ascorbic acid (AA), $\mathrm{Ag}_{n}$ nuclei were generated both on the surface and in the interior of the $\mathrm{AgCl}$ octahedra, followed by their evolution into single-crystal seeds and finally Ag nanocubes. The presence of $\mathrm{FeCl}_{3}$ as an oxidative etchant to remove the twinned seeds further facilitated the formation of Ag nanocubes in a high morphology yield. The as-synthesized Ag nanocubes showed strong SERS activity due to the preserved sharp corners and edges.

\section{Results and Discussion}

\section{Synthesis of Ag nanocubes}

Figure $1 \mathrm{~A}$ shows the transmission electron microscopy (TEM) image of a sample obtained at 
$t=6 \mathrm{~h}$ into the synthesis. The nanocubes had an average edge length of $93 \pm 4 \mathrm{~nm}$. It is worth noting that the corners and edges of these nanocubes were sharper than their counterparts prepared at a higher temperature (typically, $160{ }^{\circ} \mathrm{C}$ ) through polyol reduction. The two-dimensional array of nanocubes showed an inter-particle separation of about $6.4 \mathrm{~nm}$ (Fig. S1), twice that of the thickness of a CTAC bilayer $(\sim 3 \mathrm{~nm})$. This result indicates that CTAC formed a bilayer structure on the side faces of each Ag nanocube in the aqueous solution, making the nanocubes hydrophilic and thus well-dispersed in water. Figure 1, B and C, shows scanning transmission electron microscopy (SEM) images of the same sample at two different magnifications. For a standard synthesis, the typical morphology yield of Ag nanocubes was approximately $70 \%$, with the presence of some right bipyramids, truncated right bipyramids, and nanorods as the side products. The red curve in Figure 1D shows the UV-vis spectrum recorded from an aqueous suspension of the as-obtained nanocubes. The four well-defined LSPR peaks are consistent with the previous experimental and calculation results for Ag nanocubes with a similar size. ${ }^{28,29}$ The black curve shows the extinction spectrum obtained using discrete dipole approximation (DDA) calculation for a Ag nanocube with $2.3 \mathrm{~nm}$ truncation at the corners and $0.6 \mathrm{~nm}$ truncation at the edges, which is in good agreement with the experimental data.

\section{Growth Mechanism}

In an effort to decipher the growth mechanism, we examined the products obtained at different stages of a standard synthesis using both SEM (Fig. 2) and TEM (Fig. 3). When $\mathrm{CF}_{3} \mathrm{COOAg}$ was added into an aqueous CTAC solution, $\mathrm{AgCl}$ nanoscale octahedra were formed immediately within the first several minutes. As shown in Figure 2A, well-defined $\mathrm{AgCl}$ octahedra with an average edge length of about $150 \mathrm{~nm}$ were formed at $t=5 \mathrm{~min}$ into the synthesis. These octahedra were highly sensitive to the electron beam, suggesting a composition of AgCl rather than Ag. ${ }^{30}$ In fact, under the irradiation of electron beam, Ag nanoparticles (the bright dots) were formed at the corner and edge sites of a $\mathrm{AgCl}$ octahedron owing to the reduction of $\mathrm{AgCl}$ by the electron beam. This observation suggests the possible existence of $\mathrm{Ag}_{\mathrm{n}}$ nuclei (e.g., $\mathrm{Ag}_{2}, \mathrm{Ag}_{3}{ }^{+}$, and $\mathrm{Ag}_{4}$ among others) in the $\mathrm{AgCl}$ octahedra prior to SEM characterization.

A closer examination shows that the corners of the AgCl octahedra obtained at $t=30 \mathrm{~min}$ (Fig. 2B) were rounded. Further extension of the reaction time to $t=90 \mathrm{~min}$ and $120 \mathrm{~min}$ led to 
more significant destruction to the structure, as shown in Figure 2, C and D. These results indicate that $\mathrm{AgCl}$ was gradually reduced and dissolved as the synthesis was continued. The TEM images in Figure 3 clearly show the co-existence of $\mathrm{AgCl}$ and $\mathrm{Ag}$ nanoparticles throughout the synthesis. At $t=30 \mathrm{~min}$, the number of Ag nanoparticles was relatively small and they were mainly spherical in shape. As the reaction proceeded, the number of AgCl particles would drop while more and larger Ag nanocubes could be identified. This observation implies that most of the Ag nanocubes might be embedded in the interior of the $\mathrm{AgCl}$ nanoparticles and were later exposed due to the reduction and dissolution of $\mathrm{AgCl}$. It is worth pointing out that the $\mathrm{AgCl}$ nanoparticles could be readily dissolved by introducing additional CTAC solution. This offers us the opportunity to attain Ag nanocubes with average edge lengths of $36 \pm 3 \mathrm{~nm}$ and $56 \pm 5 \mathrm{~nm}$ (Fig. $\mathrm{S} 2$ ), respectively, for the two samples obtained at $t=90 \mathrm{~min}$ at $t=120 \mathrm{~min}$. At $t=150 \mathrm{~min}$ into the synthesis, we could still observe a small amount of AgCl mixed in the products (Fig. 3D). The AgCl component completely disappeared after 3 h, as demonstrated by the sample shown in Figure 1. Taken together, it can be concluded that both $\mathrm{Ag}_{\mathrm{n}}$ nuclei and $\mathrm{AgCl}$ nanoparticles were formed in the very early stage of a synthesis and the AgCl component then served as a precursor to elemental Ag during the evolution of the $\mathrm{Ag}_{n}$ nuclei into single-crystal seeds and then nanocubes.

The results of this time-dependent experiment indicate that the Ag nanocrystals might grow directly on the surface or inside the preformed AgCl octahedra at the expense of AgCl through continuous reduction. To validate this mechanism, we used high-resolution TEM to characterize the samples obtained in the early stage of a synthesis. At $t=60 \mathrm{~min}$, more than half of the particles were found to contain two components with different contrasts (Fig. 4A). Under the electron beam, the lighter component gradually melted and became part of the darker component. The $d$-spacing values of these two components were also different: $0.20 \mathrm{~nm}$ and 0.27 $\mathrm{nm}$ for the darker and lighter components, respectively, which could be assigned to the $d$-spacing of $\{200\}$ facets for face-centered-cubic ( $f c c)$ Ag and cubic AgCl phase (Fig. 4B), ${ }^{31}$ respectively. The HRTEM images in Figure 4, C and D, indicate that there was a rotation of $45^{\circ}$ between the two $<200>$ axes of these two single-crystal components. This observation can be understood by considering the fact that the length of face diagonal of the Ag unit cell is almost the same as the edge length of the AgCl unit cell. This correlation further explains why these two crystal phases adhere to each other despite their large difference in lattice constant. The epitaxial relationship 
between the $\mathrm{Ag}$ and $\mathrm{AgCl}$ components suggests that the $\mathrm{Ag}$ nanocube was formed by developing the Ag nucleus directly attached to the preformed $\mathrm{AgCl}$ nanoparticle.

It should be emphasized that all our experiments were carried out under the room light from fluorescent lamps. The solution typically showed a yellowish green color after $6 \mathrm{~h}$ into the standard synthesis (Fig. S3, the left vial). When the glass vial was wrapped with aluminum foil throughout the synthesis, the solution took a pale white color after heating at $60{ }^{\circ} \mathrm{C}$ for $6 \mathrm{~h}$ (Fig. $\mathrm{S} 3$, the right vial). The products mainly contained $\mathrm{AgCl}$ particles, indicating the lack of reduction from $\mathrm{AgCl}$ to Ag. This observation indicates the important role of photolysis in the formation of Ag nuclei and photo-reduction in the following step of growth.

Oxidative etching is important for the growth of metal nanocrystals with a specific internal structure because it can selectively dissolve the kinetically less favorable twinned seeds. ${ }^{32}$ Iron(III) chloride has been used as an oxidative etchant for the removal of twinned seeds and particles to facilitate the formation of single-crystal Ag nanocubes. ${ }^{33}$ In our synthesis, without the introduction of $\mathrm{FeCl}_{3}$, the percent of twinned particles in the final products became much higher (Fig. S4A). When the concentration of $\mathrm{FeCl}_{3}$ was raised to $17.16 \mu \mathrm{M}$ (four times as high as the concentration of $\mathrm{FeCl}_{3}$ used in the standard synthesis), the $\mathrm{Ag}$ nanocubes started to show severe truncations at the corners and edges due to the enhancement in oxidative etching (Fig. S4B). ${ }^{34,35}$ Taken together, a proper concentration of etchant should be used for eliminating the twinned seeds and particles during the synthesis and at the same time, keeping the corners and edges sharp.

In summary, the mechanism underlying the nucleation and growth of Ag nanocubes in this aqueous system shares a great deal in common with the photographic process. ${ }^{36}$ The nucleation of $\mathrm{Ag}_{\mathrm{n}}$ nuclei is similar to the formation of latent image centers and the further growth of these nuclei can be referred to as the development of the latent image centers. To be more specific, the synthesis starts with the formation of $\mathrm{AgCl}$ octahedra and small $\mathrm{Ag}_{\mathrm{n}}$ nuclei (Fig. 5). The nuclei could have been formed in the early stage of a synthesis due to the presence of room light and reducing agent, or even inherited from the Ag precursor. ${ }^{37,38}$ Both light and reducing agent are well-known to have the ability to generate stable $\mathrm{Ag}_{2}$ and $\mathrm{Ag}_{4}$ on $\mathrm{AgCl}$ particles. The $\mathrm{Ag}_{2}$ cluster is believed to be the smallest stable nuclei while the $\mathrm{Ag}_{4}$ is the smallest active cluster to initiate the development of Ag nanoparticles. ${ }^{37}$ Similar to the formation of latent image centers, these $\mathrm{Ag}_{\mathrm{n}}$ nuclei can be formed both on the surface and in the interior of a $\mathrm{AgCl}$ nanoparticle through 
photolysis by the room light and reduction by the ascorbic acid, preferably at the kink or defect sites of the $\mathrm{AgCl}$ nanoparticle. Compare to the $\mathrm{Ag}_{\mathrm{n}}$ nuclei positioned on the surface, the internal nuclei would be ineffective in promoting growth until the outer layer of $\mathrm{AgCl}$ has been dissolved away or reduced. ${ }^{36}$ This means the $\mathrm{Ag}_{\mathrm{n}}$ nuclei on the surface should grow first with the reduction and dissolution of $\mathrm{AgCl}$ (Fig. 5(i)) and only after the solid structure of $\mathrm{AgCl}$ octahedra is shattered, the internal nuclei would be exposed to the reducing agent and start to grow (Fig. 5(ii)).

Two types of Ag sources could be involved in the growth (development) of $\mathrm{Ag}_{n}$ nuclei: $i$ ) $\mathrm{Ag}^{+}$ions in the lattice of $\mathrm{AgCl}$ particles and ii) $\mathrm{Ag}^{+}$ions released due to the dissolution of $\mathrm{AgCl} .{ }^{36}$ The process involves the reduction of the first Ag source is known as chemical or direct development in photography, and these $\mathrm{Ag}^{+}$ions are reduced through a solid-state reaction at the $\mathrm{Ag} / \mathrm{AgCl}$ interface. The $\mathrm{Ag}^{+}$ions include those already existing or those that will diffuse to the interface through the solid $\mathrm{AgCl}$ particles. The supply of $\mathrm{Ag}^{+}$ions from this source is not adequate for attaining a cubic shape, and thus typically leads to the formation of spherical nanoparticles with a size of less than $20 \mathrm{~nm}$ and eventually Ag filaments with a diameter of 20 $\mathrm{nm}$, as reported in early studies. ${ }^{37}$ The reduction process involving the dissociated $\mathrm{Ag}^{+}$ions is known as solution physical development, which is believed to occur at the Ag/liquid interface. Considering the small surface area of $\mathrm{Ag}_{\mathrm{n}}$ nuclei, this reduction process is very slow at the early stages, but becomes dominant as the size of the Ag nanoparticles increased. ${ }^{36}$ Since this reduction occurs at the $\mathrm{Ag} /$ liquid interface, the shape of resultant Ag nanoparticles can be altered in the presence of a capping agent. In our system, the growth of $\mathrm{Ag}_{\mathrm{n}}$ nuclei mainly started from the direct development, which is confirmed by the lack of a cubic shape for the small $\mathrm{Ag}$ nanoparticles in early stages of a synthesis. Once the surface area of the Ag nanoparticles had been enlarged to a certain level, the contribution from the solution physical process would become significant. As such, the Ag nanoparticles would keep growing by self-catalyzing the reduction of free $\mathrm{Ag}^{+}$in the solution with the $\{100\}$ facets being capped by $\mathrm{Cl}^{-}$ions. This growth leads to the formation of Ag nanocubes in the final product (Fig. 5(iii)). The use of $\mathrm{FeCl}_{3}$ at an appropriate concentration is critical for selectively etching away twinned seeds and particles without influencing the sharp corners and edges. Oswald ripening might also happen throughout the synthesis, which could explain why we did not observe small nanocubes in the final products. This mechanism is completely different from what is involved in the polyol synthesis of Ag 
nanocubes. In a typical polyol process involving the introduction of $\mathrm{NaCl}$ or $\mathrm{HCl}$ to mediate the reduction kinetics, AgCl was precipitated out in the initial stage but quickly reduced to elemental Ag to facilitate the formation of seeds and then nanocubes. ${ }^{6}$ In the aqueous system, the reduction of $\mathrm{AgCl}$ and the evolution of $\mathrm{Ag}$ nanocubes seem to occur simultaneously throughout the synthesis.

Compared to the conventional polyol synthesis of Ag nanocubes, the new protocol uses $\mathrm{Cl}^{-}$ ions instead of PVP as a capping agent for the Ag $\{100\}$ facets. Chloride ion has a much smaller size than the long polymer chain of PVP, thus it can better cover the sides faces to make the edges and corners sharp. The use of a relatively low reaction temperature in the aqueous system also ensures that the diffusion of Ag atoms is slowed down and the oxidative etching on edges and corners are weakened. All these factors contribute to the formation of Ag nanocubes with sharp corners and edges.

\section{Influence of reduction kinetics}

Our synthesis involves both the reduction of $\mathrm{Ag}^{+}$ions in the solid state and the reduction of dissociated $\mathrm{Ag}^{+}$ions on the surface of $\mathrm{Ag}_{n}$ nuclei. As such, any factor capable of altering the reduction rate (including, for example, temperature, $\mathrm{pH}$ value, and the nature of precursor/reducing agent) would affect the nucleation and growth of Ag nanocubes. We first investigated the influence of temperature on the products. At room temperature, we only observed AgCl nanoparticles. When the temperature was increased to $50{ }^{\circ} \mathrm{C}$, the products were dominated by Ag nanocubes with an average edge length of $57 \pm 3 \mathrm{~nm}$ (Fig. 6A), much smaller than the nanocubes obtained using the standard protocol at $60{ }^{\circ} \mathrm{C}$ (Fig. 1A). There were also many smaller Ag nanoparticles and AgCl particles in the products. These results indicate that the weakened reducing power at a lower temperature was unable to reduce all the AgCl within 6 hours. When the temperature was increased to $70{ }^{\circ} \mathrm{C}$, the Ag nanocubes had an average edge length of $86 \pm 4 \mathrm{~nm}$ (Fig. 6B), slightly smaller than what was obtained at $60{ }^{\circ} \mathrm{C}$. The higher temperature triggered a faster reduction rate, leading to the formation of more $\mathrm{Ag}_{\mathrm{n}}$ nuclei in the nucleation stage and thus smaller size for the final products. Nevertheless, the increase in reaction temperature did not noticeably improve the percentage yield of nanocubes (still around 70\%). The as-obtained Ag nanocubes also became more truncated when the reaction temperature

was increased. Taken together, a reaction temperature around $60^{\circ} \mathrm{C}$ seems to be optimal for the 
formation of Ag nanocubes with sharp corners and edges.

The reducing power of AA is known to increase with increasing the $\mathrm{pH}$ value. ${ }^{39}$ As such, we carried out a set of experiments to figure out the relationship between the final products and the $\mathrm{pH}$ value. By introducing $\mathrm{HCl}$ or $\mathrm{NaOH}$, we could easily fine tune the $\mathrm{pH}$ value and thereby the reducing power of AA. The $\mathrm{pH}$ value of a standard synthesis was around 3.1 throughout the reaction. Figure 6, C and D, shows TEM images of the products obtained at other $\mathrm{pH}$ values. When the $\mathrm{pH}$ was set to 2.7 , the products were dominated by truncated right bipyramids. This can be ascribed to the reduced reduction rate, which favors the formation of twinned seeds. When the $\mathrm{pH}$ was increased to 7.4, the final products mainly contained irregular nanoparticles with a much smaller size than those obtained using a standard protocol. The faster reduction rate during the nucleation stage increased the number of seeds, leading to a smaller particle size when the amount of Ag precursor was fixed.

We also examined the impact of reducing agent on the final products by replacing the AA in the standard protocol with formic acid or $\mathrm{NaBH}_{4}$. The products were made of $\mathrm{AgCl}$ in the absence of an externally added reductant. When formic acid instead of AA was used, the products were still mainly composed of $\mathrm{AgCl}$ (Fig. 6E). Formic acid is known to have a weaker reducing power than $\mathrm{AA}$, which might be inadequate to reduce most of the $\mathrm{AgCl}$ to $\mathrm{Ag}$ within 6 hours. ${ }^{40}$ When switched to a much stronger reducing agent like $\mathrm{NaBH}_{4}$, all the $\mathrm{AgCl}$ nanoparticles were gone by $t=6 \mathrm{~h}$, but the final products mainly contained small particles with an irregular shape (Fig. 6F). Taken together, the use AA at a pH value around 3.1 seems to be optimal for the synthesis of Ag nanocubes in high morphological yield.

A proper concentration of $\mathrm{Cl}^{-}$ions is also important for regulating the reduction kinetics. In the current protocol, the $\mathrm{Cl}^{-}$ions were supplied by the CTAC. It is worth pointing out that the concentration of CTAC used in the standard synthesis was $20 \mathrm{mM}$, much higher than its critical micelle concentration (CMC), which is $1.62 \mathrm{mM}$ at $60{ }^{\circ} \mathrm{C}^{41}$ As such, the concentration of free $\mathrm{Cl}^{-}$ions in the reaction solution should be much lower than $20 \mathrm{mM}$. When replacing the CTAC with an equal molar amount of $\mathrm{NaCl}$ for the standard protocol, the solution was colorless after heating at $60{ }^{\circ} \mathrm{C}$ for $6 \mathrm{~h}$ (Fig. S5) and no solid products could be obtained by centrifugation at a speed as high as 12,000 rpm. This phenomenon can be explained by considering the equilibrium between $\mathrm{AgCl}$ and $\mathrm{AgCl}_{2}^{-}$: 


$$
\begin{gathered}
\mathrm{Ag}^{+}+\mathrm{Cl}^{-} \leftrightarrow \mathrm{AgCl} \\
\mathrm{AgCl}+\mathrm{Cl}^{-} \leftrightarrow \mathrm{AgCl}_{2}^{-}
\end{gathered}
$$

In comparison with $\mathrm{AgCl}$, it is much more difficult to reduce $\mathrm{AgCl}_{2}{ }^{-}$. In the case of $\mathrm{NaCl}$, the concentration of $\mathrm{Cl}^{-}$ions was much higher than that of $\mathrm{Ag}^{+}$so most of the $\mathrm{Ag}^{+}$ions were converted to the $\mathrm{AgCl}_{2}{ }^{-}$form and thus no reduction could occur. That is the reason why the solution remained clear and no solid products could be obtained at a centrifugation speed of 12,000 rpm after $6 \mathrm{~h}$. In the case of CTAC, however, the concentration of free $\mathrm{Cl}^{-}$ions was significantly lower due to the formation of micelles, making the majority of $\mathrm{Ag}^{+}$ions stay in the $\mathrm{AgCl}$ form to allow reduction by $\mathrm{AA}$. We also tested $\mathrm{NaCl}$ at lower concentrations to mimic the effect of CTAC. When the molar ratio of $\mathrm{NaCl}$ to $\mathrm{CF}_{3} \mathrm{COOAg}$ was kept at 1:1, a mixture of large, irregular and cubic nanoparticles can be readily identified in the solution (Fig. S6A), while no uniform Ag nanocubes were observed. Most of these nanoparticles were made of $\mathrm{AgCl}$, which was confirmed by the energy-dispersive X-ray spectroscopy (EDX) mapping data in Fig. S6B. This argument was also supported by the fact that most of these nanoparticles could be dissolved after soaking in $20 \mathrm{mM}$ aqueous $\mathrm{NaCl}$ solution for $3 \mathrm{~h}$. It is noteworthy that those $\mathrm{AgCl}$ nanoparticles were quite stable under the electron beam during the SEM characterization, which might be the result of different facets exposed at the surface as compared to the $\mathrm{AgCl}$ octahedra. This result indicates that, besides a proper amount of $\mathrm{Cl}^{-}$ions, the octahedral shape of the $\mathrm{AgCl}$ nanocrystals is also a key factor to the formation of Ag nanocubes, making it difficult to replace the CTAC with $\mathrm{NaCl}$.

\section{SERS activity}

The sharpness of our Ag nanocubes were further confirmed by their SERS activities (Fig. S7). We performed the measurements with 1,4-benzenedithiol (1,4-BDT) as the probe molecule and a $532 \mathrm{~nm}$ laser for excitation. The red curve shows the SERS spectrum recorded from a suspension of 93-nm Ag nanocubes, only with the introduction of 1,4-BDT adequate for the formation of a monolayer on the nanocubes. A reference solution with $0.05 \mathrm{M}$ 1,4-BDT in

aqueous $\mathrm{NaOH}$ was also measured to calculate the surface enhancement factors (EFs) of the Ag nanocubes. The EF can be calculated by using expression (3): 


$$
\mathrm{EF}=\frac{I_{\text {SERS }}}{I_{\text {Bulk }}} \times \frac{N_{\text {Bulk }}}{N_{\text {SERS }}}
$$

where $I_{\mathrm{SERS}}$ and $I_{\mathrm{Bulk}}$ are the intensities of the same band for the SERS and reference spectrum, $N_{\text {SERS }}$ is the number of adsorbed molecules probed in the SERS spectrum, and $N_{\text {Bulk }}$ is the number of molecules probed in the ordinary spectrum. We used the intensity of the peak at 1563 $\mathrm{cm}^{-1}$ to calculate the SERS enhancement factor, which gave us a value of $1.34 \times 10^{5}$. Compared to the SERS enhancement factors of 100-nm nanocubes and 90-nm nanocubes with sharp corners and edges that were prepared in a polyol system with the assistance of $\mathrm{Cl}^{-}$ions (i.e., $1.25 \times 10^{5}$ and $\left.1.26 \times 10^{5}\right)$, ${ }^{28}$ this value is compatible, confirming the presence of sharp corners and edges.

\section{Conclusions}

In summary, we have successfully demonstrated an aqueous protocol for the synthesis of Ag nanocubes with an average edge length of 35-95 nm. Upon mixing CTAC with $\mathrm{CF}_{3} \mathrm{COOAg}$ AgCl nanoscale octahedra are immediately formed. The presence of room light and a reducing agent such as ascorbic acid offers a proper reducing power to generate the $\mathrm{Ag}_{\mathrm{n}}$ nuclei and to continuously provide elemental Ag for the evolution of $\mathrm{Ag}_{\mathrm{n}}$ nuclei into Ag nanocubes. The capping of $\{100\}$ facets by $\mathrm{Cl}^{-}$ions and the use of relatively low reaction temperature are both critical to retaining the sharp corners and edges on the Ag nanocubes. This synthetic method is appealing for bio-related applications, as well as plasmonic and SERS-based sensing, due to the involvement of aqueous solutions only. It also offers a more reproducible, environmentally friendly, and economical route to the synthesis of Ag nanocubes as compared to the widely used polyol process.

\section{Experimental Section}

Chemicals and Materials: Cetyltrimethylammonium chloride (CTAC, 25 wt\% in $\mathrm{H}_{2} \mathrm{O}$ ), ascorbic acid (AA, $\geq 99.0 \%$ ), silver trifluoroacetate $\left(\mathrm{CF}_{3} \mathrm{COOAg}, \geq 99.99 \%\right)$, iron(III) chloride $\left(\mathrm{FeCl}_{3}, 97 \%\right)$, formic acid ( $\left.\geq 95 \%\right)$, sodium borohydride $\left(\mathrm{NaBH}_{4}, \geq 99 \%\right)$, sodium chloride $(\mathrm{NaCl}$, $\geq 99.0 \%$ ), and 1,4-benzenedithiol (1,4-BDT) were all purchased from Sigma-Aldrich and used as received. Deionized (DI) water with a resistivity of $18.2 \mathrm{M} \Omega \cdot \mathrm{cm}$ was used for all experiments.

Synthesis of Ag Nanocubes: In a standard protocol, aqueous solutions of CTAC (5 mL, 20 $\mathrm{mM})$ and AA (0.5 mL, $100 \mathrm{mM}$ ) were added into a $20 \mathrm{~mL}$ glass vial and heated at $60{ }^{\circ} \mathrm{C}$ for 10 
min. Aqueous solutions of $\mathrm{CF}_{3} \mathrm{COOAg}(50 \mu \mathrm{L}, 10 \mathrm{mM})$ and $\mathrm{FeCl}_{3}(80 \mu \mathrm{L}, 4.29 \mu \mathrm{M})$ were then added in one shot. The reaction solution had a $\mathrm{pH}$ value of about 3.1 during the entire synthesis. After $6 \mathrm{~h}$, the products were collected by centrifugation at 8,000 rpm and re-dispersed in DI water. For the synthesis ended at an earlier stage, the speed of centrifugation was set to 17,500 rpm and the products were re-dispersed in ethanol. All the syntheses were carried out under room light (fluorescent lamp, Sylvania Fo25/835/Eco).

SERS measurements: The SERS spectra were measured using a protocol reported before. ${ }^{28}$ To be specific, an aqueous solution of the as-obtained $\mathrm{Ag}$ nanocubes $(0.063 \mathrm{mg} / \mathrm{mL})$ and $1.2 \times 10^{-3} \mathrm{mM}$ 1,4-BDT were prepared one hour before SERS measurements. The 1,4-BDT was used at a concentration just enough to generate a monolayer on the Ag nanocubes by assuming a footprint of $0.54 \mathrm{~nm}^{2}$. The reference solution was prepared by dissolving 1,4-BDT in cooled 12 $\mathrm{M}$ aqueous $\mathrm{NaOH}$ one hour prior to the measurement. The Raman spectra were recorded using a Renishaw inVia Raman spectrometer coupled with a Leica microscope using a $100 \times$ objective. The excitation wavelength was $532 \mathrm{~nm}$, and the holographic notch filter has a grating of 2400 lines/mm. Data was collected from the solution phase with a collection time of $30 \mathrm{~s}$ at $50 \mathrm{~mW}$ for all the samples.

Characterization methods: The TEM images were taken using a Hitachi HT7700 microscope operated at $120 \mathrm{kV}$. The SEM images and EDX mapping were taken using a Hitachi SU8230 microscope. UV-vis extinction spectra were recorded on a Cary 60 spectrometer (Agilent Technologies, Santa Clara, CA). High-resolution TEM (HRTEM) images were acquired using JEOL 2100F microscope (JEOL, Tokyo, Japan) operated at $200 \mathrm{kV}$. The $\mathrm{pH}$ values were measured using a Fisher Scientific AB15 pH meter. SERS spectra were taken using a Renishaw inVia micro Raman system (Renishaw, Hoffman Estates, IL). Discrete dipole approximation (DDA) calculation was carried out using DDSCAT 7.3.

\section{ASSOCIATED CONTENT}

\section{Supporting Information}

The Supporting Information is available free of charge on the ACS Publications website.

TEM image of the as-synthesized Ag nanocubes and a plot of the transmitted electron intensity;

TEM images of Ag nanocubes obtained in the early stages of a synthesis and the corresponding UV-vis spectra; photograph of reaction solutions for standard syntheses conducted with room 
light and in the dark; TEM images of $\mathrm{Ag}$ nanostructures obtained using $\mathrm{FeCl}_{3}$ with different amounts; photograph of a reaction solution obtained by substituting CTAC with an equal molar amount of NaCl; SEM images and EDX mapping of samples obtained by substituting CTAC with $\mathrm{NaCl}$ at the same molar amount as $\mathrm{CF}_{3} \mathrm{COOAg}$; and SERS spectra.

\section{AUTHOR INFORMATION}

\section{Corresponding Author}

*E-mail: younan.xia@bme.gatech.edu

\section{Notes}

The authors declare no competing financial interest.

\section{ACKNOWLEDGEMENTS}

This work was supported in part by a grant from NSF (DMR-1506018) and startup funds from the Georgia Institute of Technology. As a jointly supervised Ph.D. candidate from Shandong University, J.L. was also partially supported by a fellowship from the China Scholarship Council. 


\section{References}

(1) Rycenga, M.; Cobley, C. M.; Zeng, J.; Li, W.; Moran, C. H.; Zhang, Q.; Qin, D.; Xia, Y. Controlling the Synthesis and Assembly of Silver Nanostructures for Plasmonic Applications. Chem. Rev. 2011, 111, 3669-3712.

(2) Zhang, Q.; Li, W.; Moran, C.; Zeng, J.; Chen, J.; Wen, L.-P.; Xia, Y. Seed-Mediated Synthesis of Ag Nanocubes with Controllable Edge Lengths in the Range of 30-200 nm and Comparison of Their Optical Properties. J. Am. Chem. Soc. 2010, 132, 11372-11378.

(3) Liang, H.; Wang, W.; Huang, Y.; Zhang, S.; Wei, H.; Xu, H. Controlled Synthesis of Uniform Silver Nanospheres. J. Phys. Chem. C 2010, 114, 7427-7431.

(4) Sun, Y.; Xia, Y. Shape-Controlled Synthesis of Gold and Silver Nanoparticles. Science 2002, 298, 2176-2179.

(5) Siekkinen, A. R.; McLellan, J. M.; Chen, J.; Xia, Y. Rapid Synthesis of Small Silver Nanocubes by Mediating Polyol Reduction with a Trace Amount of Sodium Sulfide or Sodium Hydrosulfide. Chem. Phys. Lett. 2006, 432, 491-496.

(6) Im, S. H.; Lee, Y. T.; Wiley, B.; Xia, Y. Large-Scale Synthesis of Silver Nanocubes: The Role of $\mathrm{HCl}$ in Promoting Cube Perfection and Monodispersity. Angew. Chemie 2005, 44, 2192-2195.

(7) Wang, Y.; Zheng, Y.; Huang, C. Z.; Xia, Y. Synthesis of Ag Nanocubes 18-32 nm in Edge Length: The Effects of Polyol on Reduction Kinetics, Size Control, and Reproducibility. J. Am. Chem. Soc. 2013, 135, 1941-1951.

(8) Ruditskiy, A.; Xia, Y. Toward the Synthesis of Sub-15 nm Ag Nanocubes with Sharp Corners and Edges: The Roles of Heterogeneous Nucleation and Surface Capping. J. Am. Chem. Soc. 2016, 138, 3161-3167.

(9) Wiley, B. J.; Chen, Y.; McLellan, J. M.; Xiong, Y.; Li, Z.-Y.; Ginger, D.; Xia, Y. Synthesis and Optical Properties of Silver Nanobars and Nanorice. Nano Lett. 2007, 7, 1032-1036.

(10) Zhou, J.; An, J.; Tang, B.; Xu, S.; Cao, Y.; Zhao, B.; Xu, W.; Chang, J.; Lombardi, J. R. Growth of Tetrahedral Silver Nanocrystals in Aqueous Solution and Their SERS Enhancement. Langmuir 2008, 24, 10407-10413. 
(11) Wang, Y.; Wan, D.; Xie, S.; Xia, X.; Huang, C. Z.; Xia, Y. Synthesis of Silver Octahedra with Controlled Sizes and Optical Properties via Seed-Mediated Growth. ACS Nano 2013, 7, 4586-4594.

(12) Pietrobon, B.; Kitaev, V. Photochemical Synthesis of Monodisperse Size-Controlled Silver Decahedral Nanoparticles and Their Remarkable Optical Properties. Chem. Mater. 2008, 20, 5186-5190.

(13) Wiley, B. J.; Xiong, Y.; Li, Z.-Y.; Yin, Y.; Xia, Y. Right Bipyramids of Silver: A New Shape Derived from Single Twinned Seeds. Nano Lett. 2006, 6, 765-768.

(14) Sun, Y.; Mayers, B.; Herricks, T.; Xia, Y. Polyol Synthesis of Uniform Silver Nanowires: A Plausible Growth Mechanism and the Supporting Evidence. Nano Lett. 2003, 3, 955960.

(15) Skrabalak, S. E.; Au, L.; Li, X.; Xia, Y. Facile Synthesis of Ag Nanocubes and Au Nanocages. Nat. Protoc. 2007, 2, 2182-2190.

(16) Yang, X.; Yang, M.; Pang, B.; Vara, M.; Xia, Y. Gold Nanomaterials at Work in Biomedicine. Chem. Rev. 2015, 115, 10410-10488.

(17) Cobley, C. M.; Skrabalak, S. E.; Campbell, D. J.; Xia, Y. Shape-Controlled Synthesis of Silver Nanoparticles for Plasmonic and Sensing Applications. Plasmonics 2009, 4, 171179.

(18) Lim, B.; Jiang, M.; Tao, J.; Camargo, P. H. C.; Zhu, Y.; Xia, Y. Shape-Controlled Synthesis of Pd Nanocrystals in Aqueous Solutions. Adv. Funct. Mater. 2009, 19, 189200.

(19) Zeng, J.; Zheng, Y.; Rycenga, M.; Tao, J.; Li, Z.-Y.; Zhang, Q.; Zhu, Y.; Xia, Y. Controlling the Shapes of Silver Nanocrystals with Different Capping Agents. J. Am. Chem. Soc. 2010, 132, 8552-8553.

(20) Lin, Z.-W.; Tsao, Y.-C.; Yang, M.-Y.; Huang, M. H. Seed-Mediated Growth of Silver Nanocubes in Aqueous Solution with Tunable Size and Their Conversion to $\mathrm{Au}$ Nanocages with Efficient Photothermal Property. Chem. - Eur. J. 2016, 22, 2326-2332. 
(21) Ma, Y.; Li, W.; Cho, E. C.; Li, Z.-Y.; Yu, T.; Zeng, J.; Xie, Z.; Xia, Y. Au@Ag Core-Shell Nanocubes with Finely Tuned and Well-Controlled Sizes, Shell Thicknesses, and Optical Properties. ACS Nano 2010, 4, 6725-6734.

(22) Samal, A. K.; Polavarapu, L.; Rodal-Cedeira, S.; Liz-Marzán, L. M.; Pérez-Juste, J.; Pastoriza-Santos, I. Size Tunable Au@Ag Core-Shell Nanoparticles: Synthesis and Surface-Enhanced Raman Scattering Properties. Langmuir 2013, 29, 15076-15082.

(23) Gong, J.; Zhou, F.; Li, Z.-Y.; Tang, Z. Controlled Synthesis of Non-Epitaxially Grown Pd@Ag Core-Shell Nanocrystals of Interesting Optical Performance. Chem. Commun. 2013, 49, 4379-4381.

(24) Zhu, C.; Zeng, J.; Tao, J.; Johnson, M. C.; Schmidt-Krey, I.; Blubaugh, L.; Zhu, Y.; Gu, Z.; Xia, Y. Kinetically Controlled Overgrowth of Ag or Au on Pd Nanocrystal Seeds: From Hybrid Dimers to Nonconcentric and Concentric Bimetallic Nanocrystals. J. Am. Chem. Soc. 2012, 134, 15822-15831.

(25) Yu, D.; Yam, V. W.-W. Controlled Synthesis of Monodisperse Silver Nanocubes in Water. J. Am. Chem. Soc. 2004, 126, 13200-13201.

(26) Yu, D.; Yam, V. W.-W. Hydrothermal-Induced Assembly of Colloidal Silver Spheres into Various Nanoparticles on the Basis of HTAB-Modified Silver Mirror Reaction. J. Phys. Chem. B 2005, 109, 5497-5503.

(27) Yin, Y.; Li, Z.-Y.; Zhong, Z.; Gates, B.; Xia, Y.; Venkateswaran, S. Synthesis and Characterization of Stable Aqueous Dispersions of Silver Nanoparticles through the Tollens Process. J. Mater. Chem. 2002, 12, 522-527.

(28) McLellan, J. M.; Siekkinen, A.; Chen, J.; Xia, Y. Comparison of the Surface-Enhanced Raman Scattering on Sharp and Truncated Silver Nanocubes. Chem. Phys. Lett. 2006, 427, 122-126.

(29) Hung, L.; Lee, S. Y.; McGovern, O.; Rabin, O.; Mayergoyz, I. Calculation and Measurement of Radiation Corrections for Plasmon Resonances in Nanoparticles. Phys. Rev. B 2013, 88, 075424. 
(30) Schuette, W. M.; Buhro, W. E. Silver Chloride as a Heterogeneous Nucleant for the Growth of Silver Nanowires. ACS Nano 2013, 7, 3844-3853.

(31) Zhu, Y.; Liu, H.; Yang, L.; Liu, J. Study on the Synthesis of Ag/AgCl Nanoparticles and Their Photocatalytic Properties. Mater. Res. Bull. 2012, 47, 3452-3458.

(32) Long, R.; Zhou, S.; Wiley, B. J.; Xiong, Y. Oxidative Etching for Controlled Synthesis of Metal Nanocrystals: Atomic Addition and Subtraction. Chem. Soc. Rev. 2014, 43, 62886310.

(33) Ma, Y.; Li, W.; Zeng, J.; McKiernan, M.; Xie, Z.; Xia, Y. Synthesis of Small Silver Nanocubes in a Hydrophobic Solvent by Introducing Oxidative Etching with Fe(III) Species. J. Mater. Chem. 2010, 20, 3586.

(34) Li, W.; Camargo, P. H. C.; Au, L.; Zhang, Q.; Rycenga, M.; Xia, Y. Etching and Dimerization: A Simple and Versatile Route to Dimers of Silver Nanospheres with a Range of Sizes. Angew. Chemie 2010, 49, 164-168.

(35) Cobley, C. M.; Rycenga, M.; Zhou, F.; Li, Z.-Y.; Xia, Y. Controlled Etching as a Route to High Quality Silver Nanospheres for Optical Studies. J. Phys. Chem. C 2009, 113, 1697516982.

(36) James, T. H. The Theory of the Photographic Process. New York Macmillan, 1977.

(37) Tani, T. Silver Nanoparticles: From Silver Halide Photography to Plasmons; Oxford University Press, 2015.

(38) Chang, S.; Chen, K.; Hua, Q.; Ma, Y.; Huang, W. Evidence for the Growth Mechanisms of Silver Nanocubes and Nanowires. J. Phys. Chem. C 2011, 115, 7979-7986.

(39) Yang, Y.; Liu, J.; Fu, Z.-W.; Qin, D. Galvanic Replacement-Free Deposition of Au on Ag for Core-Shell Nanocubes with Enhanced Chemical Stability and SERS Activity. J. Am. Chem. Soc. 2014, 136, 8153-8156.

(40) Shi, L.; Wang, A.; Zhang, T.; Zhang, B.; Su, D.; Li, H.; Song, Y. One-Step Synthesis of Au-Pd Alloy Nanodendrites and Their Catalytic Activity. J. Phys. Chem. C 2013, 117, $12526-12536$. 
(41) El Abdi, R.; Rujinski, A. D.; Poulain, M. Effects of Immersion Duration and Temperature on Mechanical Properties of Optical Fibers Aged in CTAC Aqueous Solution. Eng. Sci. Technol. An Int. J. 2015, 18, 52-58. 

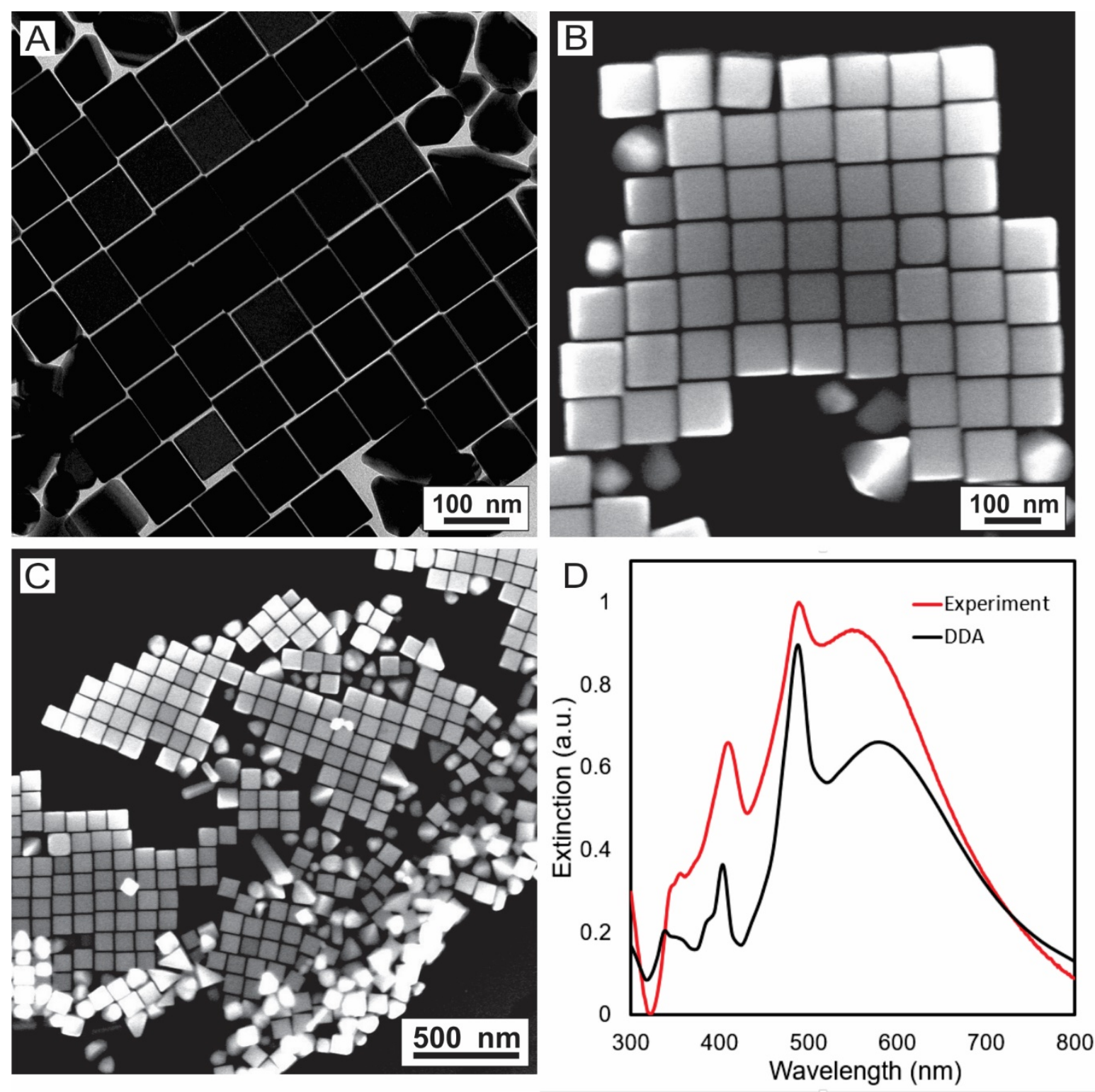

Figure 1. A) TEM and B) SEM images of the as-synthesized Ag nanocubes with an average edge length of $93 \pm 4 \mathrm{~nm}$. C) A low-magnification SEM image of the Ag nanocubes, showing the co-existence of Ag nanocrystals with other shapes. D) UV-vis spectrum recorded from an aqueous suspension of the sample shown in (C) and the DDA calculation result for a $93 \mathrm{~nm}$ cube with $2.3 \mathrm{~nm}$ truncation at the corners and $0.6 \mathrm{~nm}$ truncation at the edges. 

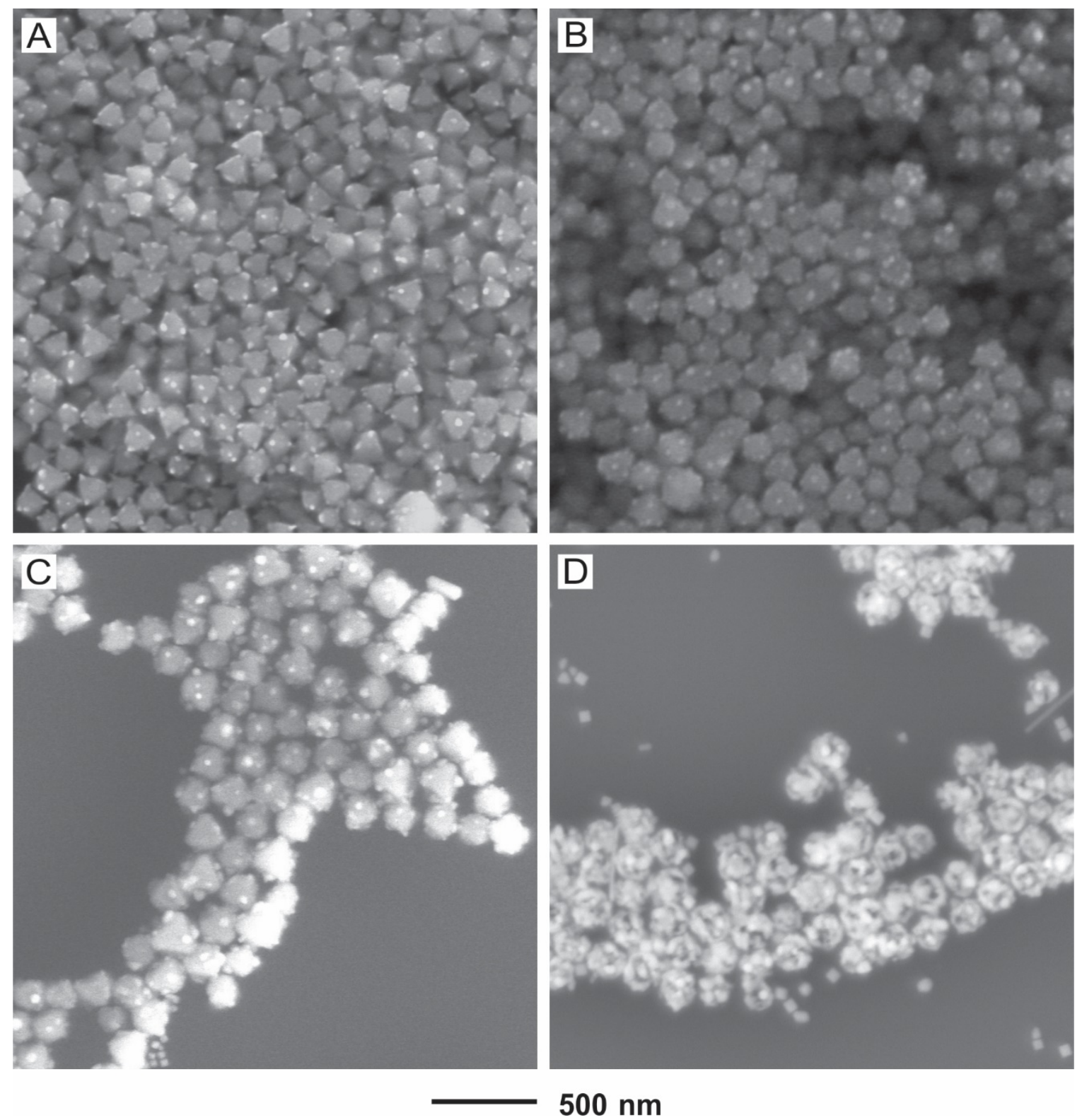

Figure 2. SEM images of samples obtained at different stages of a standard synthesis: A) 5, B) 30, C) 90 and D) 120 min. The octahedral nanoparticles shown in (A-D) were made of AgCl while the small, bright dots can be assigned to Ag nanoparticles formed from the decomposition of AgCl upon exposure to electron beam (typically within a few seconds). Silver nanoparticles with a cubic shape could be easily identified in (C-D). 

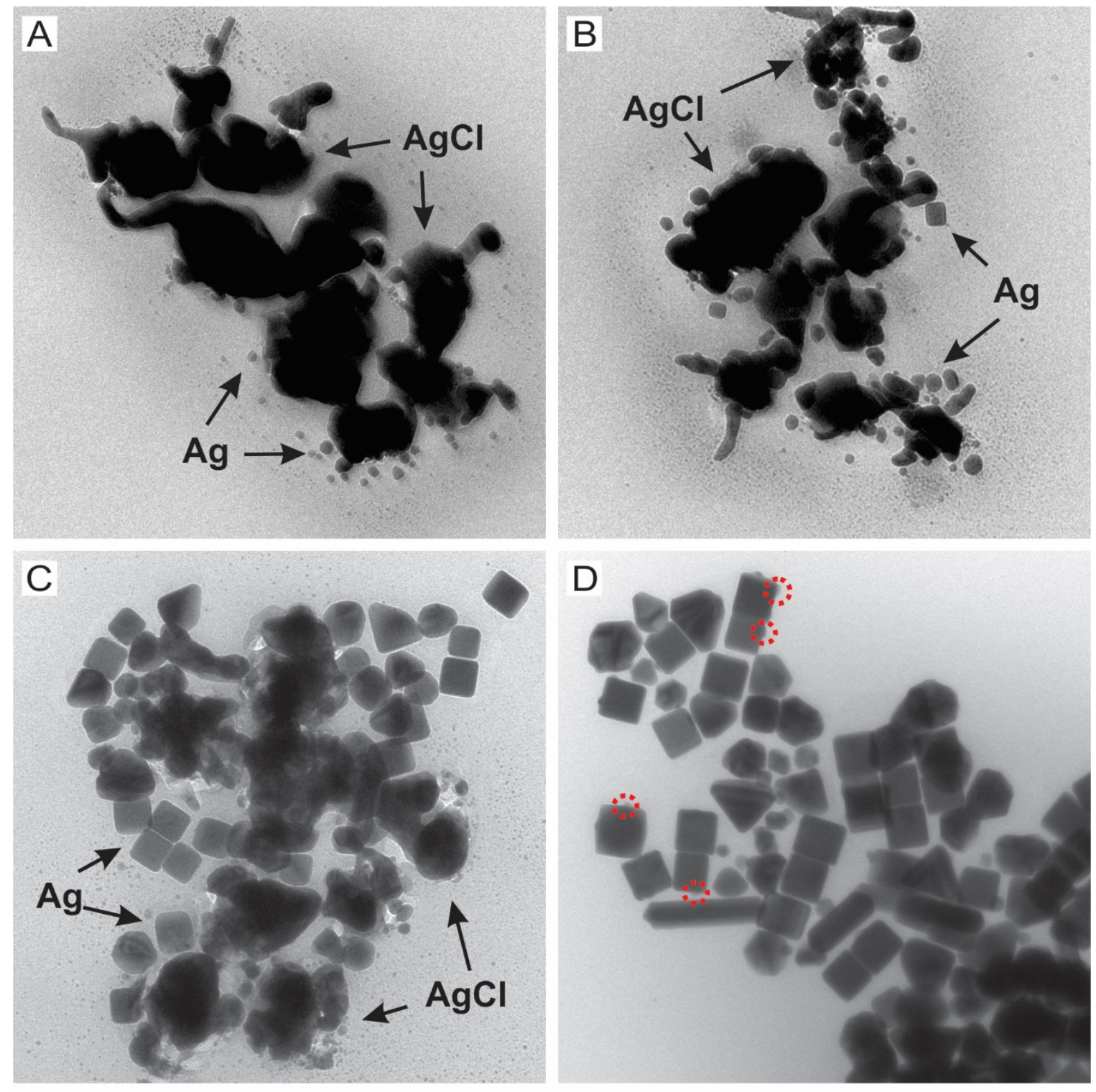

\section{$100 \mathrm{~nm}$}

Figure 3. TEM images of samples obtained at different stages of a standard synthesis: A) 30, B) 90, C) 120 and D) 150 min. The big irregular AgCl particles and Ag nanoparticles were indicated by arrows in (A-C). A very small amount of $\mathrm{AgCl}$ can still be observed on the surface of some Ag nanocubes in (D), as marked by red dotted circles. 

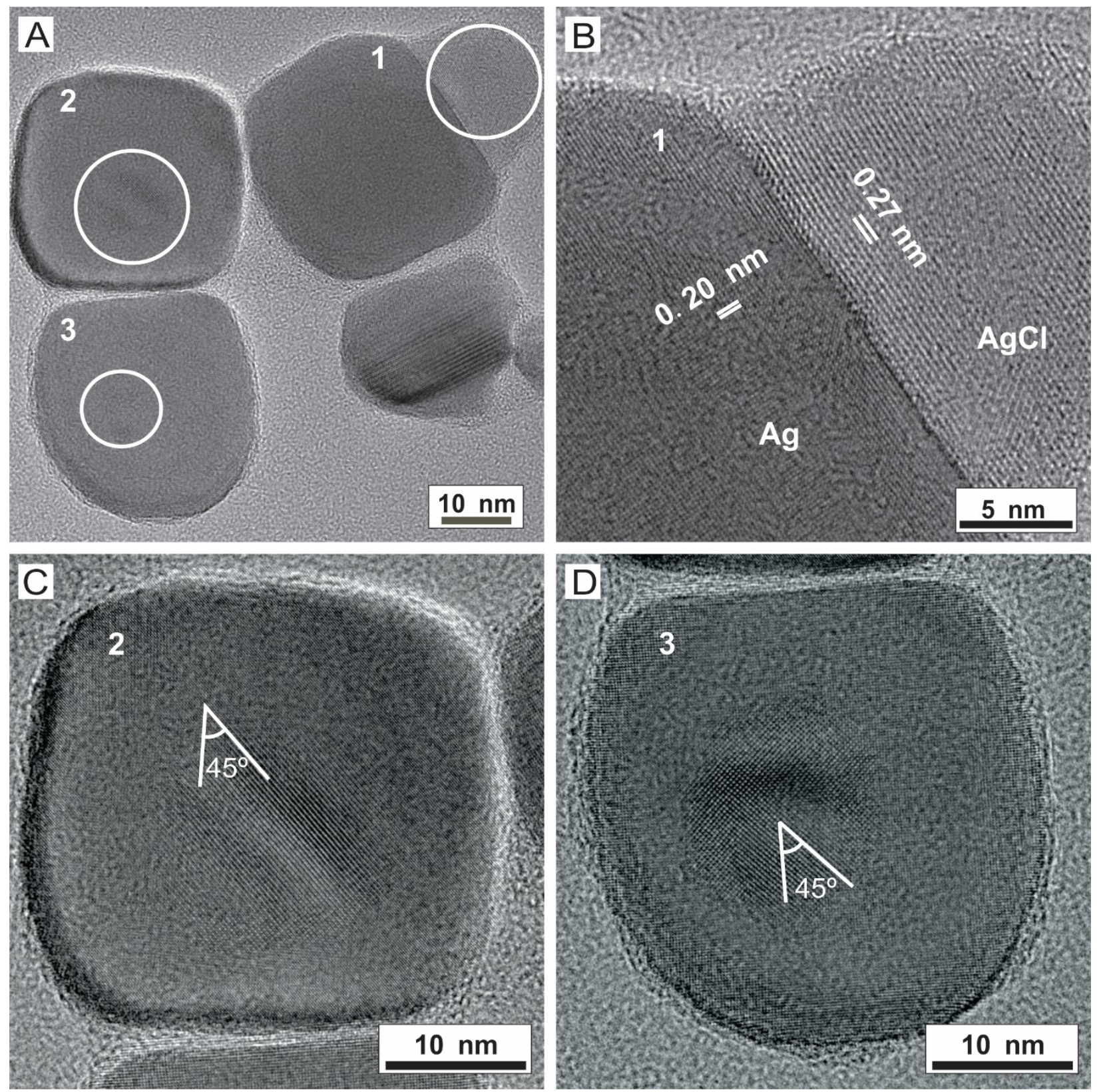

Figure 4. A) HRTEM image of Ag nanocubes obtained at $t=60 \mathrm{~min}$. The circles indicate the presence of AgCl on three Ag nanocubes (labelled with 1, 2, and 3). B) Magnified HRTEM image taken from the interface region between Ag nanocube 1 and $\mathrm{AgCl}$ components. C, D) HRTEM image of Ag nanocubes 2 and 3 with AgCl components on top of their $\{100\}$ facets. There was a $45^{\circ}$ angle between the $<200>$ axes of Ag and AgCl lattices. 


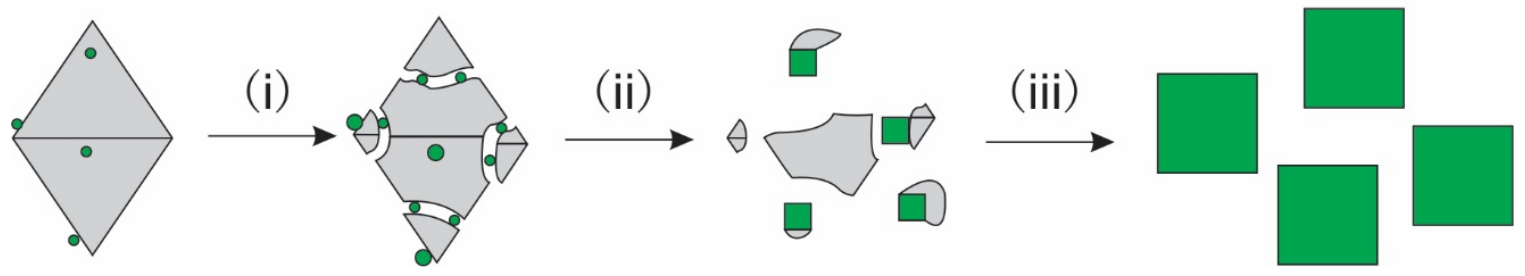

Figure 5. Schematic of the mechanism involved in the formation of Ag nanocubes in an aqueous system. The AgCl and Ag are shown in grey and green colors, respectively. The synthesis can be divided into three stages: i) Formation of AgCl octahedra upon mixing $\mathrm{CTAC}$ with $\mathrm{CF}_{3} \mathrm{COOAg}$ Small $\mathrm{Ag}_{n}$ nuclei are formed both on the surface and in the interior of AgCl octahedra as a result of exposure to light and/or reducing agent. When the $\mathrm{Ag}_{\mathrm{n}}$ nuclei on the surface start to grow, the underlying AgCl octahedron will start to disappear due to reduction and dissolution. ii) Exposure of the internal $\mathrm{Ag}_{n}$ nuclei to the reducing environment. All the $\mathrm{Ag}_{\mathrm{n}}$ nuclei quickly grow in size at the expense of remaining $\mathrm{AgCl}$. In this stage, one can observe Ag nanocubes epitaxially connected with AgCl. iii) Formation of Ag nanocubes with sharp corners and edges. 

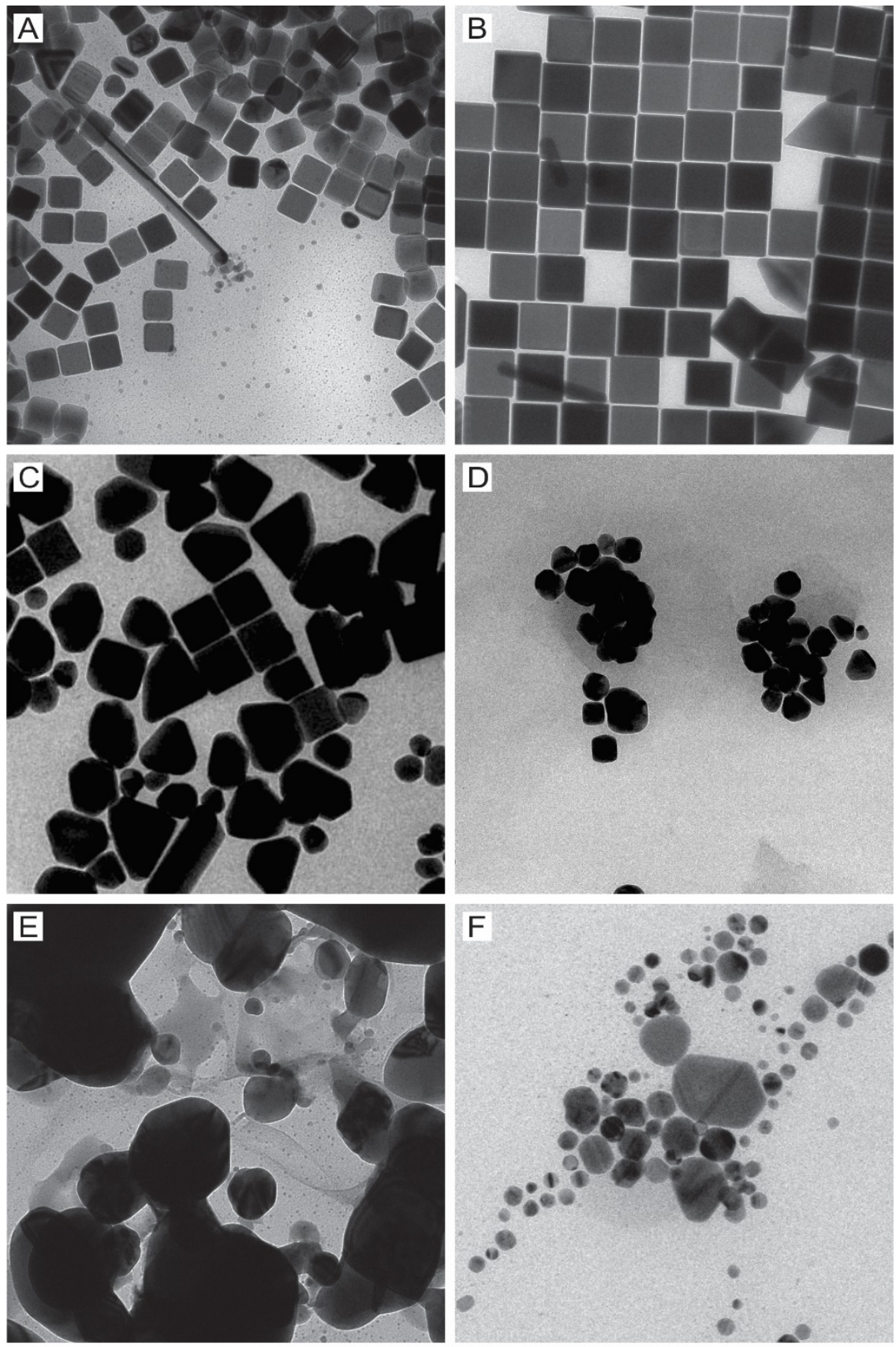

$100 \mathrm{~nm}$

Figure 6. TEM images of samples obtained under different reduction kinetics achieved through variation of reaction temperature: A) $50{ }^{\circ} \mathrm{C}$ and B) $70{ }^{\circ} \mathrm{C}$; variation of $\mathrm{pH}$ value: C) 2.7 and D) 7.4; or variation of reducing agent: E) formic acid and F) $\mathrm{NaBH}_{4}$. All other conditions were kept the same as the standard protocol and the products were collected at $t=6 \mathrm{~h}$ into the synthesis. 
TOC
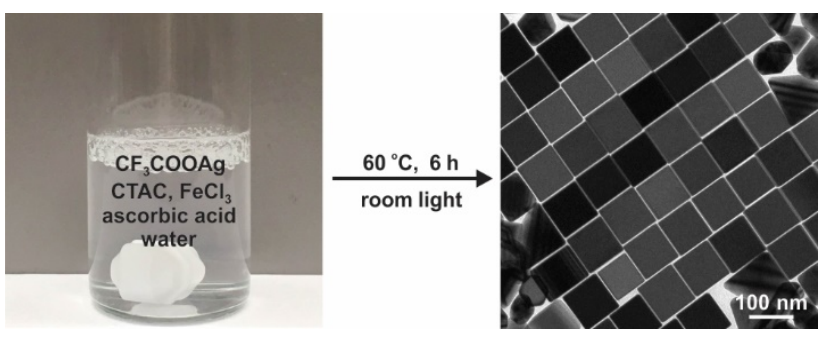